M.C. Concepcion Sales

${ }^{I}$ Philippine General Hospital, Manila, Philippines macarmelamd@yahoo.com

Primary CNS Lymphoma (PCNSL) is an unusual extranodal form of Non-Hodgkin's Lymphoma with a locally aggressive course but a rare tendency to disseminate systemically. There are various modalities available for the treatment of PCNSL which include chemotherapy, radiotherapy, surgery and immunotherapy. The effectiveness of adding another anti-neoplastic agent to HD-MTX have been optimized in small scale studies yet the " perfect combination" has yet to be elucidated Objectives: This study aims to 1) compare the response to treatment of monotherapy with highdose Methotrexate (HD-MTX) versus HD-MTX and an additional anti-neoplastic agent by evaluating complete response, partial response, stable disease and disease progression and 2) to compare the hematologic and non-hematologic side effects among patients subjected to monotherapy vs combination chemotherapy. Methodology: Journals from Medline, EMBASE, Cochrane Central Register of Control Trials (CENTRAL) and other relevant websites (www.clinicaltrials.org) without any restrictions in the year, language and status of publication were searched. Literatures cited by eligible studies and systemic reviews were also checked to identify useful articles. The following Medical Subject Headings (MeSH) were used: 'primary CNS lymphoma', 'treatment', 'chemotherapy' and 'randomized control trial'. Statistical analysis was performed using the RevMan software version 5.1. Odds ratio (OR) and $95 \%$ confidence interval $(95 \%$ CI) were used as summary statistics. Results and Conclusion: The use of high-dose methotrexate and another anti-neoplastic agent showed benefit in terms of achieving complete response and delaying disease progression among patients diagnosed with PCNSL. However, the risks of hematologic toxicities such as anemia, neutropenia, thrombocytopenia and infection was higher in patients treated with the combination chemotherapy. Significant non-hematologic side effects such as mucositis was also observed in patients receiving an add-on to high dose methotrexate.

PC3 - 138

doi:10.1017/cjn.2016.391

\section{Stereotactic Radiosurgery for Intracranial} Hemangiopericytomas - A Multicenter Study

O. Cohen-Inbar ${ }^{1,2,3}$

${ }^{1}$ Department of Neurological Surgery, Rambam Health Care Center, Haifa Israel

${ }^{2}$ Molecular Immunology Laboratory, Technion Israel Institute of Technology

${ }^{3}$ Department of Neurosurgery and Gamma-Knife Center, University of Virginia, Charlottesville, Virginia oc2f@virginia.edu

Hemangiopericytomas (HPC) are widely recognized for their aggressive clinical behavior. We report a large multicenter study, through the International Gamma Knife Research Foundation reviewing management and outcome following stereotactic radiosurgery (SRS) for recurrent or newly-discovered HPC's. Methods: Eight centers participated, reviewing a total of 90 patients harboring 133 tumors. Prior treatments included embolization $(\mathrm{n}=8)$, chemotherapy $(\mathrm{n}=2)$, and fractionated radiotherapy $(n=34)$. The median tumor volume at the time of SRS was $4.9 \mathrm{ml}$ (range 0.2-42.4 ml). WHO-grade II (typical) HPC's formed $78.9 \%(\mathrm{n}=71)$ of the cohort. The median margin and maximal doses delivered were 15 Gy (2.8-24) and 32 Gy (8-51), respectively. The median clinical and radiographic follow-up period was 59 months (6-190) and 59 months (6-183), respectively. Results: At last follow-up, $55 \%$ of tumors and $62.2 \%$ of patients demonstrated local tumor control. New remote intracranial tumors were found in $27.8 \%$. 24.4\% of patients developed extra-cranial metastases. Adverse radiation effects were noted in $6.7 \%$. The overall survival was $91.5 \%, 82.1 \%, 73.9 \%, 56.7 \%$, and $53.7 \%$ at 2 , $4,6,8$, and 10 years, respectively, after initial SRS. Local progression free survival was $81.7 \%, 66.3 \%, 54.5 \%, 37.2 \%$, and $25.5 \%$ at $2,4,6,8$, and 10 years, respectively, after initial SRS. In our cohort, 32 patients underwent 48 repeat SRS procedures for 76 lesions. Margin dose greater than $16 \mathrm{~Gy}(\mathrm{p}=0.037)$ and tumor histology $(\mathrm{p}=0.006)$ were shown to influence PFS. Conclusions: SRS provides a reasonable rate of local tumor control and a low risk of adverse effects.

\section{PC3 - 141}

doi:10.1017/cjn.2016.392

\section{Long-Term Results of Stereotactic Radiosurgery for Skull Base Meningiomas}

O. Cohen-Inbar ${ }^{1,2,3}$

${ }^{1}$ Department of Neurological Surgery, Rambam Health Care Center, Haifa Israel

${ }^{2}$ Molecular Immunology Laboratory, Technion Israel Institute of

Technology

${ }^{3}$ Department of Neurosurgery and Gamma-Knife Center,

University of Virginia, Charlottesville, Virginia

oc2f@virginia.edu

Gamma knife radiosurgery (GKRS) is well-established in the management of inaccessible, recurrent, or residual benign skull base meningiomas. Most series report clinical outcome parameters and complications in the short -intermediate period after radiosurgery. Reports of long-term tumor control and neurological status are still lacking. Objective: We report the presentation, treatment, and long-term outcome of skull base meningiomas after GKRS. Methods: From a prospectively collected IRB approved database, we selected patients with a WHO grade I skull base meningioma treated with a single-session GKRS and a minimum of 60 months follow up. 135 patients, $54.1 \%$ males $(n=73)$ form the cohort. Median age was 54 years (19-80). Median tumor volume was $4.7 \mathrm{~cm} 3(0.5-23)$. Median margin dose was $15 \mathrm{~Gy}$ (7.5-36). Median follow up was 102.5 months (60.1-235.4). Patient and tumor characteristics were assessed to determine predictors of neurological function and tumor progression. Results: At last follow up, tumor volume control was achieved in $88.1 \%(n=119)$. Post-GKRS clinical improvement or stability was reported in $61.5 \%$. The 5,10 , and 15 years actuarial progression free survival rates are $100 \%, 95.4 \%$, and $68.8 \%$, respectively. Favorable outcome (both tumor control and clinical preservation/improvement) was attained in $60.8 \%(\mathrm{n}=79)$. PreGKRS performance status (KPS) was shown to influence tumor progression $(\mathrm{p}=0.0001)$ and post-GKRS clinical improvement / preservation $(\mathrm{p}=0.003)$. Conclusion: GKRS offers a highly durable rate of tumor control for WHO-I skull base meningiomas, with an acceptably low incidence of neurological deficits. KPS at the time of radiosurgery serves as a reliable long-term predictor of overall outcome. 